\title{
Editorial
}

\section{Klug entscheiden}

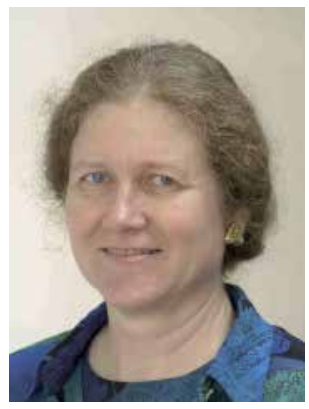

„Als Ärzte ist es unsere Pflicht zu behandeln, aber auch Behandlungen zu unterlassen, wenn sie dem Patienten nichts nützen oder sogar schaden könnten", sagte Kongresspräsident Prof. Dr. Michael Hallek auf dem 121. Kongress der Deutschen Gesellschaft für Innere Medizin (DGIM) in Mannheim. Mit der Kampagne „Klug entscheiden“, die sich an die in den USA sehr erfolgreiche Kampagne „Choosing Wisely" anlehnt, in der es um das Vermeiden von Überdiagnostik und Übertherapie geht, will die DGIM mehr Bewusstsein für das Problem schaffen.

Im Rahmen dieses Kongresses hielt Prof. Giovanni Maio, Direktor des Instituts für Ethik und Geschichte der Medizin an der Albert-Ludwigs-Universität Freiburg, am 18. April 2015 in einem stark überfüllten Saal einen begeistert aufgenommenen Vortrag zum Thema „Die ärztliche Kunst des Unterlassens“. Er führte darin aus, dass der Patient das Handeln des Arztes in der Situation der Unsicherheit als etwas Beruhigendes und Erleichterndes empfindet. Nach der ärztlichen Berufsethik muss das Handeln, zu dem auch die Beratung gehört, angemessen sein. Nach G. Maio ist das Beratungsgespräch die Hauptleistung des Arztes. Es kann jedoch nur dann qualitativ hochwertig sein, wenn er die Zeit und die Ressourcen hat, die Komplexität der Patientengeschichte zu durchdringen. Hat er das jeweilige Patientenproblem erkannt, muss er die vorhandene Evidenz einschließlich der persönlichen Erfahrung auf die individuelle und unverwechselbare Krankengeschichte anwenden, um die bestmögliche Lösung zu erreichen. Leitlinien und Algorithmen bieten dabei nur ein grobes Gerüst für die Einzelfallentscheidung. Die Beratung stellt somit eine enorme kreative Integrationsleistung des Arztes dar. Erst danach sollten, mög- lichst in gemeinsamer Entscheidung mit dem Patienten, Maßnahmen im Sinne der Behandlung ergriffen werden. Eine Verschwendung, d.h. Behandlungen, die dem Patienten nichts nützen oder ihm sogar schaden können, muss dabei vermieden werden - die „Kunst des Unterlassens“.

G. Maio führte weiter aus, dass die im Beratungsgespräch aufgebaute Arzt-Patienten-Beziehung bekanntlich die Grundlage ärztlichen Handelns darstellt. Im zunehmend ökonomisierten deutschen Gesundheitssystem, das vor allem der Anbieter-orientierten Akutmedizin gerecht wird, wird jedoch umso mehr bezahlt und erstattet, je mehr Patienten durchgeschleust werden und je mehr "getan“ wird. Dies trägt erheblich zum rasanten Kostenanstieg der modernen Medizin bei. Die erfahrungsgesättigte Beratung des Arztes wird dagegen nur minimal vergütet, d.h. sie wird nach der aktuell herrschenden Finanzlogik nicht wertgeschätzt. Infolge des Wirtschaftlichkeitsgebotes im Sinne einer Gewinnmaximierung und infolge des Konkurrenzdrucks werden insbesondere die für die Anbieter finanziell attraktiven Behandlungen auch zunehmend beworben. Den Patienten wird dadurch suggeriert, dass über die technischen und pharmakologischen Möglichkeiten der Medizin jedes Problem ohne seine gezielte Mitarbeit am Gesundungsprozess lösbar sei.

Bei der modernen Medizinindustrie stehen somit Produktionsgesichtspunkte, Beschleunigung und Effizienz im Vordergrund. Die Ärzte sollen hingegen auf den Aspekt eines Leistungserbringers reduziert werden und objektivierbare und messbare Sachleistungen erbringen, d.h. es werden Bewertungskriterien über sie verhängt, die mit ihrer eigentlichen ärztlichen Aufgabe und mit ihrer ärztlichen 
Kernleistung nichts zu tun haben. Die mittlerweile geforderte „Ergebnisqualität“ könnte, so G. Maio, eventuell dazu führen, dass man sich vor allem auf Patienten konzentriert, mit denen gute „Outcomes“ zu erreichen sind. Patienten mit komplexen und schwierigen Krankheitsbildern oder mit chronischen und unheilbaren Erkrankungen könnten dagegen die positive Leistungsstatistik gefährden.

Die tradierten Prioritäten und die Kultur der Medizin haben sich somit sukzessive verändert. Die Folgen sind - so G. Maio - schon deutlich sichtbar: Die Ärzte sind in die Machbarkeitsfalle geraten und stehen damit unter einem enormen $\mathrm{Er}$ wartungsdruck. Sie befinden sich zudem in einem Loyalitätskonflikt, denn sie haben eine Loyalitätspflicht gegenüber den Patienten, deren Wohl an oberster Stelle stehen muss. Andererseits wird seitens der Unternehmen, für die sie tätig sind, Aktion, d.h. (Be)handlung unter Berücksichtigung von Rentabilitätsgesichtspunk- ten gefordert. Die für den korrekten Entscheidungsprozess erforderliche qualitativ hochwertige ärztliche Beratung passt jedoch nicht zum geforderten Aktionismus, insbesondere dann nicht, wenn der Arzt auf die Grenzen des Machbaren verweisen muss. Er befindet sich hier jedoch nicht nur in der Defensive, sondern muss sich eventuell sogar mit Regressforderungen von Patienten auseinandersetzen, weil für klärende Gespräche, in denen auch die Zweifel und Unsicherheiten in der Medizin dargestellt werden, nicht genügend Zeit zur Verfügung stand.

G. Maio fordert deshalb einen Paradigmenwechsel, d.h. dass dem einzelnen Arzt wieder die Freiheit gegeben werden muss, die vorhandenen Ressourcen im Sinne des Patienten zu nutzen. Insbesondere sollte es Anreize für die Ärzte geben, ihre Patienten gut zu beraten im Sinne des „klug entscheiden“. Er fordert ein neues Verständnis von ärztlicher Leistung und für Qualität ein, denn der Arzt- beruf ist ein Beruf der Zuwendung und der Fürsorge.

Was hat dies alles mit Phytotherapie zu tun? Die Phytotherapie ist ein gutes Beispiel für all diejenigen in der Regel preiswerten Therapieverfahren, die eine aktive Mitwirkung des Patienten erfordern, die nicht dem modernen Medizin-Aktionismus entsprechen bzw. die sich der Gewinnmaximierungsstrategie widersetzen, weil sie eine intensive Beratung und/oder Zuwendung zum Patienten erfordern. Es hat eine gewisse Logik, dass in diesem System viele dieser Verfahren ohne Berücksichtigung von Wirkungs- und Wirksamkeitsnachweisen schon vor längerer Zeit als „medizinisch nicht notwendig“ und damit als Verschwendung von Ressourcen klassifiziert wurden.

Ich wünsche Ihnen viel Freude beim Lesen dieses Heftes.

\section{Karin Kraft}

Online

http://dx.doi.org/10.1055/s-0041-102855 